\title{
Frontières
}

\section{Recherche et pratique en prévention du suicide}

\section{François Chagnon}

Volume 21, numéro 1, automne 2008

Prévenir le suicide

URI : https://id.erudit.org/iderudit/037868ar

DOI : https://doi.org/10.7202/037868ar

Aller au sommaire du numéro

Éditeur(s)

Université du Québec à Montréal

ISSN

1180-3479 (imprimé)

1916-0976 (numérique)

Découvrir la revue

Citer ce document

Chagnon, F. (2008). Recherche et pratique en prévention du suicide. Frontières, 21(1), 8-9. https://doi.org/10.7202/037868ar d'utilisation que vous pouvez consulter en ligne.

https://apropos.erudit.org/fr/usagers/politique-dutilisation/ 


\section{RECHERCHE ET PRATIQUE EN PRÉVENTION DU SUICIDE}

\author{
François Chagnon, Ph. D., \\ directeur du numéro.
}

En 2009, l'Institut national de santé publique du Québec confirmait une baisse marquée des suicides au Québec. Ainsi, «depuis le sommet de 22 décès par 100000 personnes atteint en 1999, le taux de suicide a diminué en moyenne de 4,1\% par année» (Gagné et St-Laurent, 2009). Cette baisse, bien qu'elle concerne également le suicide des femmes, serait principalement attribuable à une diminution importante des suicides chez les jeunes hommes.

Cette bonne nouvelle n'est certes pas une victoire définitive ou totale puisque les taux de suicides peuvent fluctuer et que malgré cette baisse marquée, les taux de suicide au Québec demeurent élevés en comparaison de ceux relevés dans d'autres provinces canadiennes ou pays. Il s'agit toutefois d'un gain extrêmement important pour la prévention du suicide ainsi que pour toutes les personnes touchées par le suicide. Chaque mort évitée est une victoire pour la vie.

Une fois cette nouvelle accueillie avec soulagement, après des années de hausses sévères des suicides, se pose implacablement la recherche des explications : à quoi devons-nous attribuer la diminution des taux de suicide au Québec? Formuler une réponse à cette question nous oblige à beaucoup de prudence. En effet, malgré les avancées de la connaissance en prévention du suicide, il n'existe pas de recherches qui démontrent les causes de ce fléchissement des taux de suicide. Cela, en raison de la complexité inhérente au phénomène du suicide, mais aussi parce qu'il existe différentes approches théoriques, chacune mettant en cause des niveaux d'explications et des variables différentes pouvant causer le suicide (Mishara et Tousignant, 2004).

Ainsi, selon la théorie psychanalytique formulée par Freud au début du $20^{\mathrm{e}}$ siècle, le suicide tire son origine de conflits au sein du psychisme des individus et notamment de l'état de mélancolie subséquent. Dans cette même logique, Menninger avancera l'hypothèse d'une tendance à l'autodestruction chez les humains afin d'expliquer le suicide.

Différemment, selon le sociologue Émile Durkheim, les causes du suicide résident davantage dans la société que chez les individus eux-mêmes. Il faut donc examiner les forces sociales, ses mouvements et la place qu'occupent les individus afin de comprendre le suicide. Cette dernière explication, bien qu'elle soit incomplète et qu'elle ne permette pas de prédire le suicide dans toutes les sociétés, demeure d'un grand intérêt du fait qu'elle porte à examiner le rôle de la société et des facteurs psychosociaux dans le suicide.

Les théories psychologiques plus contemporaines examinent le rôle des traits de personnalité dans le suicide. Selon Edwin
Shneidman (cité dans Mishara et Tousignant, 2004), trois principaux facteurs réunis dans un agencement singulier contribuent au suicide: une intense souffrance psychologique, un état de perturbation et un contexte de pression extrême. Le suicide apparaît dès lors comme l'unique solution afin d'échapper à la souffrance.

Selon l'approche psychiatrique, les maladies caractérisées que sont les troubles mentaux sont la principale cause des suicides. Les troubles affectifs, l'abus ou la dépendance aux drogues et à l'alcool, ainsi que la concomitance entre les différents troubles y joueraient un rôle prépondérant. Enfin, les recherches médicales récentes analysent comment les facteurs biologiques et génétiques peuvent accroître la vulnérabilité et le risque de suicide, notamment l'impulsivité et les prédispositions à la dépression.

Ces théories ne représentent qu'une partie des explications possibles du suicide. Bien qu'elles se fondent sur des hypothèses différentes et parfois contradictoires, on relève que la communauté scientifique s'entend généralement pour reconnaître que le suicide est un phénomène multidéterminé et que divers facteurs de risque allant des prédispositions individuelles aux causes sociales doivent être pris en considération afin de comprendre et de prévenir le suicide. Ce numéro spécial de la revue Frontières propose une pluralité de points de vue et de recherches sur le suicide tout en mettant l'accent sur des interventions et des expériences de partenariat entre la recherche et la pratique.

La section article s'ouvre avec un article de Michel Hanus qui nous propose une réflexion sur le deuil après suicide, ses conséquences et ses mécanismes de résolution possibles. On y aborde notamment la spécificité du deuil par suicide qui, encore de nos jours, est entourée de stigma et de tabous. Hanus y traite aussi avec beaucoup de finesse de la délicate question du deuil après suicide chez les jeunes et les enfants et du rôle des accompagnants.

Dans son article intitulé «De la prévention du suicide comme une question sociologique», Matthieu Lustman nous interpelle sur la question «le suicide est-il encore un problème moral? ». L'évolution de la recherche scientifique a-t-elle pour effet de nous détourner de la question du sens du suicide et, si c'est le cas, quelles en sont les conséquences pour la prévention du suicide? À partir d'une approche sociologique, Lustman cherche à comprendre les valeurs implicites de la prévention et ensuite à savoir si la prévention diffère d'un pays à l'autre en raison des facteurs socioculturels. Il s'intéresse de ce fait à la construction sociale du suicide.

L'article de Marie Julien et Jean-Jacques Breton aborde la question de l'efficacité de la prévention du suicide chez les adolescents à partir d'une revue systématique de 11 différentes stratégies de prévention. L'article propose une analyse critique fondée sur la 
base théorique des programmes, le moment de l'intervention, le choix des populations, le partage des responsabilités et les problèmes liés à la recherche évaluative. En conclusion, les auteurs discutent des principaux défis et opportunités afin d'améliorer la prévention du suicide chez les jeunes.

Catherine Laurier examine la question du suicide chez les jeunes délinquants ou chez les jeunes présentant des troubles de la conduite. Bien que les recherches plus récentes montrent pour ces jeunes un risque particulièrement élevé de suicide, il existe encore de nos jours une croyance erronée que les délinquants sont moins à risque de suicide. L'article se fonde sur une recension critique des écrits et propose un cadre théorique afin de comprendre les facteurs de risque de suicide chez les délinquants et d'orienter la prévention du suicide auprès de ce groupe de jeunes.

Le premier article de la section Recherche présente une analyse récente de l'évolution des taux de suicide au Québec de 1981 à 2005. À cette fin, Matthieu Gagné et Danielle St-Laurent utilisent une nouvelle méthode d'analyse des taux pour déceler avec plus de précision les fluctuations entre les périodes. Cette analyse détaillée prend en considération le sexe, l'âge et la région géographique afin de comparer les taux relevés et de mieux comprendre les changements.

On estime qu'un suicide affecte profondément au moins six personnes dans l'entourage immédiat de la personne décédée. En se fondant sur une recherche réalisée auprès de 141 professionnels en santé mentale pratiquant au Québec et ayant vécu le suicide d'un patient, Melissa Henry et ses collègues examinent les impacts de ces événements sur les professionnels. La recherche analyse de façon différenciée les conséquences des suicides selon le genre des professionnels et discute des résultats en prenant appui sur les théories de la socialisation et du développement professionnel afin de dégager des pistes pour la prévention auprès des intervenants ayant la charge de patients suicidaires.

Il est reconnu que les personnes ayant reçu un diagnostic de schizophrénie ont un risque élevé de suicide. On comprend mal toutefois quel rôle joue le rapport à la maladie dans l'augmentation de ce risque. En adoptant une méthode fondée sur l'autopsie psychologique, Georgia Vrakas a comparé 29 patients décédés par suicide et un groupe de 33 patients non suicidaires ayant un diagnostic de schizophrénie ou de trouble schizo-affectif afin de tenter de mieux comprendre le rapport entre le suicide, l'acceptation de la maladie et le rôle des événements stressants. Dans cette recherche, Georgia Vrakas porte une attention particulière à l'implication de la famille et des proches afin de soutenir l'acceptation de la maladie et prévenir le suicide.

À partir de l'examen de lettres d'adieu de jeunes qui se sont suicidés, et en adoptant une méthode d'analyse qualitative, Francine Gratton et Christine Genest ont essayé de mieux comprendre ce que des adolescents et des jeunes suicidés veulent que leur suicide produise. Les auteures rapportent cinq types d'effets: des effets positifs pour soi, produits par soi ou par les autres; des effets positifs pour les autres, produits par soi ou par les autres; finalement, des effets négatifs pour les autres. À partir de ces analyses, Gratton et Genest proposent des pistes de prévention du suicide chez les jeunes.

Bien que différentes études montrent que la victimisation sexuelle augmente de façon importante le risque de suicide et de comportements suicidaires, les connaissances précises susceptibles de guider la pratique des intervenants dans le traitement de ces jeunes sont limitées. L'article de Brabant, Chagnon et Hébert présente les résultats d'une recherche menée auprès d'adolescentes en centre jeunesse ayant dévoilé une agression sexuelle. La recherche examine plus particulièrement le rôle des mécanismes d'adaptation dans la diminution des séquelles des agressions.
Malgré la productivité croissante de la recherche, il existe un écart entre la production et l'utilisation des connaissances. La section Intervention présente en ouverture les résultats d'une expérience fondée sur l'utilisation d'une communauté de pratique afin de soutenir l'application des connaissances. Cette expérience pilote menée par Chagnon et ses collègues réunissait des chercheurs et des représentants d'une quinzaine d'organisations en prévention du suicide. À partir d'une stratégie structurée et fondée sur l'intensité des échanges entre la recherche et la pratique, le projet visait à augmenter la réceptivité, l'accès aux connaissances scientifiques et leur utilisation.

Bien que rare, le suicide chez les enfants est une problématique préoccupante. Très peu de programmes et de stratégies visant la prévention du suicide chez les enfants ont été documentés. En produisant deux articles complémentaires, l'un abordant l'expérience pratique (Gallagher et Daigle) et l'autre, l'évaluation empirique (Daigle et Gallagher), Gallagher et Daigle présentent un programme de prévention du suicide auprès des enfants en milieu scolaire primaire, ainsi que les résultats de l'évaluation d'implantation du programme. Les facteurs facilitant le développement de tels programmes sont relevés et des recommandations pour leur mise en œuvre sont formulées sur la base des résultats observés.

Les autochtones sont les groupes chez lesquels les taux de suicide sont les plus élevés au Canada. Toutefois, très peu de recherches portent spécifiquement sur le suicide au sein de cette population. Dans son article, Michel Tousignant relate, en tant que directeur, l'expérience de son équipe de recherche multidisciplinaire sur la prévention du suicide en milieu autochtone. L'auteur décrit les défis et les obstacles rencontrés dans le développement du programme de recherche de son équipe. À titre d'illustration, il présente l'exemple concret d'une recherche réalisée par son équipe. En discussion, Michel Tousignant aborde la capacité à répondre aux besoins de la famille autochtone contemporaine.

Les médias de masse sont-ils responsables des suicides en grappes? Plusieurs recherches se sont intéressées à cette question et la plupart concluent à une augmentation des suicides à la suite des diffusions médiatiques. Mais qu'en est-il de la force de la preuve et quels sont les mécanismes en cause? Dans cet article, Louise Pouliot présente d'abord une synthèse critique des recherches sur le lien entre la diffusion dans les médias de masse de reportages sur les suicides et la survenue subséquente de suicides en grappes. L'auteure discute avec finesse des hypothèses courantes pour expliquer ce lien et propose des avenues afin d'améliorer la recherche en ce domaine.

Enfin, dans la section Point de vue, Charles Bedwani examine les questions qui suivent: avec l'émergence de ce qu'il est convenu d'appeler le «village global», assistons-nous depuis quelques décennies à une telle accélération de l'évolution sociale que notre psyché aurait de la peine à lui emboîter le pas, et que le processus inné de son développement en serait affecté... La souffrance des jeunes se voit-elle accrue par ces changements sociétaux? L'auteur nous propose une réflexion passionnante sur les nouveaux changements sociaux, les sentiments d'insécurité qui s'en dégagent et la réactivation d'une violence fondamentale archaïque de survie chez les adolescents.

\section{Bibliographie}

GAGNÉ, M. et D. ST-LAURENT (2009). La mortalité par suicide au Québec: tendances et données récentes - 1981 à 2007, Institut national de santé publique du Québec. <http://www.inspq.qc.ca/ publications $/$ notice $\cdot$ asp $? \mathrm{E}=\mathrm{p} \&$ NumPublication $=890>$.

MISHARA, B. L. et M. TOUSIGNANT (2004). Comprendre le suicide, Montréal, Presses de l'Université de Montréal. 